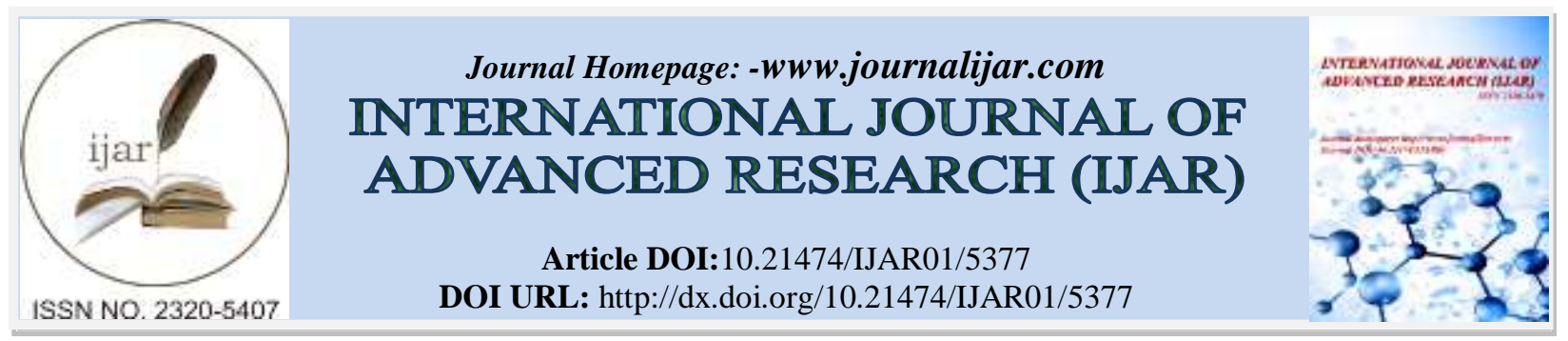

RESEARCH ARTICLE

\title{
A NEW DESIGN TITANIUM MINIPLATE VERSUS CONVENTIONAL 4-HOLE TITANIUM MINIPLATE FOR MANAGEMENT OF MANDIBULAR ANGLE FRACTURES: A COMPARATIVE STUDY.
}

\author{
Dr. Saikrishna Degala, Dr. Asutosh Das, Dr. Sujeeth Shetty, Dr. Praveen Kumar Singh and Dr. Vijay Singh \\ Rai.
}

Manuscript Info
Ma.......................
Manuscript History
Received: 09 July 2017
Final Accepted: 11 August 2017
Published: September 2017

\section{Abstract}

Copy Right, IJAR, 2017,. All rights reserved.

\section{Introduction:-}

Orofacial trauma surgery is the foundation from which the speciality of maxillofacial surgery arose, and has significantly expanded and developed over the last 50 years [2].The mandible is the largest and strongest facial bone, by virtue of its position on the face and its prominence; it is commonly fractured when maxillofacial trauma has been sustained [3]. The causes of fracture of the mandible are chiefly road traffic accidents, interpersonal violence, falls, sports injuries and industrial trauma [4]. Mandibular angle fractures account for 23-42\% of all cases of mandibular fractures. The frequent involvement can be attributed to changes in the lines of calcification and strength from horizontal body to vertical ascending ramus, the thinner cross-sectional area, and the presence of impacted or partially erupted third molars. In addition, the mandibular angle is subjected to heavy muscle forces [10]. Fractures of the mandibular angle represent an important clinical challenge because their treatment is plagued with the highest post-surgical complication rate of all mandibular fractures. Not all mandibular fractures require operative treatment, but all successful treatment of mandible fractures depends on undisturbed healing in the correct anatomic position under stable conditions. Failure to achieve these conditions of healing results in infection, malocclusion, nonunion, or malunion [6-9]. Mandibular angle fractures are associated with the highest complication rates of all mandibular fractures, yielding an incidence as high as 32\% [12-15]. Various techniques for treatment of mandibular angle fractures have been described in the literature; open reduction with non-rigid fixation by means of trans-osseous wires, cicum-mandibular wires, or small positional bone plates; AO reconstruction plates; dynamic compression plates; mini-dynamic compression plates; lag screws; and non-compression plates. Optimal treatment for angle fractures remains controversial. Various plate and screw systems that are used for treatment of mandibular angle fractures have been evaluated [11]. This in-vivo study was done to compare the results of two methods of fixation (open reduction and internal fixation) of mandibular angle fractures i.e., comparative evaluation between new design titanium miniplate and standard 4-hole titanium miniplate.

\section{Materials and Methods:-}

This in-vivo study was done on patients with angle fracture at the department of Oral and Maxillofacial Surgery. Informed consent was taken from all the patients prior to surgery and ethical committee permission was obtained. 
Patients were randomly selected as per inclusion and exclusion criteria, regardless of gender, requiring open reduction and internal fixation of mandibular angle fracture with or without other associated fractures of facial skeleton. Selection criteria included dentulous patients above 18 years of age with no significant medical history. Patients with non-comminuted, non-complicated, and minimally displaced unilateral or bilateral mandibular angle fractures alone, or fractures associated with other facial fractures were included in the study.

However, patients with systemic bone disease like osteoporosis, osteopetrosis, edentulous mandible, comminuted and infected mandibular angle fractures were excluded from the study.

Complete history was recorded, patients were examined clinically and explained about the procedure, its complications and the follow-up period involved in the study in a language that they fully comprehended. Patients complying with the inclusion criteria were planned for open reduction and internal fixation under general anesthesia. Routine blood investigations (required for pre-anesthetic evaluation), radiological investigations (OPG/PA view Mandible/Chest X-ray PA view), ECG was done prior to surgery. All the subjects received intravenous antibiotics from the time of admission until discharge.

A total of 20 patients were selected randomly and divided into following 2 groups:

Group I:-

(10 patients) - New design titanium miniplate used for fixation of mandibular angle fracture.

\section{Group II:-}

(10 patients) - Standard 4-hole titanium miniplate used for fixation of mandibular angle fracture.

Clinical parameters and radiographic findings were recorded. Relevant clinical parameters were assessed preoperatively, intraoperatively, and postoperatively.

\section{Surgical procedure:-}

Group I (New Design Titanium Miniplate):-

All cases were treated under general anesthesia with naso-endotracheal intubation. In all patients fractures were reduced with upper and lower Erich's arch bar fixation as a means for intermaxillary fixation intaoperatively. All arch bars were removed 4 weeks postoperatively.

In all cases, intraoral vestibular incision was placed. The incision was made over the external oblique ridge, beginning midway up the anterior border of the ascending ramus, and was carried into the vestibule just lateral to the first molar. Then subperiosteal dissection was performed, exposing the lateral surface of the mandibular angle and ramus region extending to the posterior and inferior border to have adequate release of the tissue to have visualization of the fracture [5].

In few of the cases, along with intraoral incision, a small stab incision was made through cutaneous surface, $1 \mathrm{~cm}$ above the inferior border of the mandible. The trocar was placed through the stab incision.

After reduction of the fracture, the straight section of the new design $2 \mathrm{~mm}$ 6-hole non-compression titanium miniplate, which has four holes, was adapted to the superior border of the external oblique ridge. Two lateral extensions, with one hole each, were then bent over to adapt to the buccal cortex of the ascending ramus along the external oblique ridge. The miniplate was secured using six 2.0 x $6-\mathrm{mm}$ monocortical titanium screws. Intraoral surgical site was closed with 3-0 vicryl and stab incision was closed in layers with 3-0 vicryl and skin incision was closed with 3-0 silk suture/ prolene 4-0.

\section{Group II (Conventional 4-Hole Titanium miniplate):-}

The surgical procedure is similar to that done for group I cases till the reduction of fracture fragments.

After reduction of the fracture, a straight $2 \mathrm{~mm}$ 4-hole with gap noncompression titanium miniplate was adapted to the superior border of the external oblique ridge. The miniplate was secured using four $2.0 \times 6-\mathrm{mm}$ monocortical titanium screws. Intraoral surgical site was closed with 3-0 vicryl.

Postoperatively all patients were given intravenous antibiotics and analgesics followed by 5 day course of oral antibiotics. Patients of both groups were kept on elastic intermaxillary fixation for 2 weeks postoperatively on an 
average. All cases have been followed up for minimum of 2 months to maximum of 12 months. Initially patients were followed up on weekly basis for the first month, then once in 15 days for the next 2 months, then once in 3 months.

All the subjects were evaluated clinically and radiologically for following parameters:-

Type of fracture: Favourable or unfavourable (both horizontally and vertically). Assessed with OPG, P.A View of mandible and intraoperative clinical examination.

Fracture segment mobility: Assessed clinically and intraoperatively.

Occlusal discrepancies: The changes in occlusion were assessed preoperatively and postoperatively over six weeks. The occlusion was scored as follows:-

Normal occlusion/ functional occlusion.

Moderate derangement - reasonable but not exact contact bilaterally.

Gross derangement - no contact or contact in one or two teeth or open bite.

Need for the intermaxillary fixation and its duration.

Paresthesia / Neurosensory changes. (Preoperative and postoperative)

Neurosensory testing was done in quiet room with the patient and examiner relaxed and comfortable. Tests were performed with the patient's eye closed. The subject indicated detection of a stimulus to the examiner by raising a finger. The results of each test were compared with those obtained from the normal (unoperated / uninjured) side.

Neurosensory dysfunctions were assessed using the following simple tests:

Light Touch Sensation:-

This test was performed using a wisp of cotton wool. An area of unpleasant sensation if present was mapped applying the stimulus within this area and then moving it outward in small steps until a sensation is felt. This position was marked on the skin and the test was repeated at a series of different sites until the region is outlined. The area of the skin mapped out was taken as the baseline value and during subsequent follow-up the values were compared and postoperative recovery analyzed.

\section{Brush directional strokes:-}

Brush stroke direction was examined using fine camel hairbrushes. The test site (The lower lip and chin on the operated side) was stroked from right to left or from left to right for a $1 \mathrm{~cm}$ length. The patient had to correctly identify the direction in 12 of 15 times or it was recorded as decreased sensibility.

Anatomic reduction: Assessed with postoperative radiographs - OPG and P.A mandible.

Good: No visible radiographic gap between fracture fragments postoperatively.

Fair: Less than $5 \mathrm{~mm}$ of visible radiographic gap between fracture fragments postoperatively.

Satisfactory: More than $5 \mathrm{~mm}$ of visible radiographic gap between fracture fragments postoperatively.

Infection at the surgical site: Assessed by any swelling, pain, tenderness, wound dehiscence or pus discharge at the operated site.

Malunion / nonunion: Assessed with relevant postoperative radiographs.

Need for the removal of the plates and screws: In case of severe or recurrent infection at the fracture site, or if the plate / screws become loose or dislodged were managed with antibiotic therapy and plate removal and scored as: 
No

2) Yes

\section{Results:-}

A total of 20 patients were included in the study out of which 8 cases (40\%) were in the age group of 18-25 years, 7 cases (35\%) in age group of 26-35 years and 5 cases (25\%) in age group of 36-45yrs with a mean age of 31.5 years. In the present study there were 17 males $(85 \%)$ and 3 females $(15 \%)$ included in both the groups with a male:female ratio of $6: 1$.

Out of the total number of subjects included in this study, $5(50 \%)$ patients in Group I \& $3(30 \%)$ patients in Group II sustained injury due to road traffic accidents. 4 (40\%) cases in Group I \& $5(50 \%)$ cases in Group II sustained injury due to self-fall. 1 case $(10 \%)$ in Group I \& 2 cases $(20 \%)$ in Group II sustained injury due to assault.

All the patients had unilateral mandibular angle fractures in association with or without other fractures of the maxillofacial skeleton. It was found that the left side mandibular angle (55\%) was more involved than the right side (45\%).

There were 6 cases $(30 \%)$ of isolated mandibular angle fracture in both the groups (3 cases - 30\% in each). Associated parasymphysis fracture was noted in 7 cases $(35 \%)$, body fracture in 4 cases $(20 \%)$ \&symphysis fracture in 1 case $(5 \%)$. Association of midface fracture was found to be in 1 case $(5 \%)$.

Fracture segment mobility was assessed intra-operatively and it was found that 10 cases (100\%) in Group I \& 8 cases $(80 \%)$ in Group II had fracture segment mobility, whereas it was found to be nil in 2 cases (20\%) in Group II.

Out of the total number of subjects 6 cases (30\%) had paresthesia preoperatively as well as post operatively, which was transient and recovered over a period of 4-6 weeks. 14 patients (70\%) did not exhibit any neurosensory changes.

Anatomic reduction was rated as good, fair or satisfactory with the help of postoperative radiographs. In this study anatomically good reduction was achieved in 8 cases $(80 \%)$ of Group I \& 7 cases (70\%) of Group II. Anatomic reduction was found to be fair in 2 cases (20\%) of Group I \& 3 cases (30\%) of Group II.

Occlusion and need for intermaxillary fixation (IMF) was found to be deranged in all the cases of both the groups. Functional occlusion was attained postoperatively with the help of IMF elastics, which was retained for a mean period of 1.57 weeks \& 1.43 weeks for Group I \& Group II patients respectively. Infection at surgical site was evident in 1 case $(10 \%)$ in each of the two groups. One of the two cases was managed with antibiotic therapy but in one case of Group I with left angle fracture, there was recurrent infection at the surgical site and it was managed with plate removal under local anesthesia and antibiotic therapy.

None of the cases in this study exhibited malunion or nonunion of fracture segments on postoperative radiographic assessment.

\section{Discussion:-}

Orofacial trauma surgery is the foundation from which the speciality of maxillofacial surgery arose, and has significantly expanded and developed over the last 50 years [2].

Managing fractures of most bones implies following a general formula i.e., recognition of the existing problem, followed by reduction of the fracture(s), retention of the bony segments in reduced position, and rehabilitation during and after bony healing [18].

Fractures of the mandible are common in patients who sustain facial trauma. Haug et al. showed a 6:2:1 proportion among mandibular, zygomatic, and maxillary fractures. Infection, malunion, malocclusion, and neurosensory dysfunction are the most common complications of mandibular fractures and their treatment [19]. The angle of mandible is commonly associated with fractures, because of 1) the presence of third molars; 2) a thinner cross- 
sectional area than the tooth bearing region; and 3) biomechanically the angle can be considered a "lever" area [5]. Treatment of angle fractures is plagued by the highest complication rates among mandible fractures, and no consensus exists regarding optimal treatment [19].

In our study of 20 patients, there was high male predominance to female with ratio of $6: 1$, which correlated to the study of Edward Ellis III [6]. The finding that the highest incidence of trauma occurred in the 20-30 year old age group is in agreement with the report from other countries [20,21]. In the series of studies conducted by Brown J.S. et al.[22], he reported that assault was the main etiological factor for mandibular fractures comprising $61.6 \%$ of the total cases. But in this present study self-fall $(45 \%)$ was found to be the major mode of injury, followed by road traffic accident (40\%), and assault (15\%). These differences may be due to the socioeconomic status of the population involved, the unemployment rates and usage of motor vehicles. According to studies conducted by Nell H. Lyuk [1], in case of injuries due to assault, the left side is more commonly involved because in most cases the assailants are right handed and the left side of the jaw is more likely to be struck. These findings are in agreement with our present series of study. It was found that left side mandibular angle was more commonly involved in the present study. The percentage of fractures caused by sports was found to be nil when compared to the studies conducted by Allan B.P et al. who reported a higher percentage of (19\%) for sports related injuries [20]. The association of parasymphysis fractures with mandibular angle fractures was found to be higher (35\%) compared to other maxillofacial fractures. Segmental mobility of the fracture fragments was checked by digital palpation. Mobility of the fragments was present in 10 patients (100\%) of Group I and 8 patients (80\%) of Group II intraoperatively before reduction. During the follow-up period, no mobility of the fragments was noted in any of the patients included in this study.

The placement of screws in the canal and entrapment of the nerve in the fracture site are two principle modes of nerve injury [23]. Monocortical plates and screws do not usually cause sensory disturbance [24]. The displacement of fracture fragments was noted and it was displaced vertically in majority of the cases in our study. It was also noted that there was a significant correlation between the preoperative displacement of the fracture fragments and the postoperative paresthesia. A similar conclusion was drawn in the series of studies conducted by Jose M.Marchena et al.[17] whereas Tateyukilizuka et al.[16] argued that the preoperative fracture displacement corelated with the preoperative sensory disturbance and had no co-relation with the postoperative paresthesia. In our study transient paresthesia was noted in 4 cases (40\%) in group I and 2 cases (20\%) in group II, who had preoperative paresthesia which gradually improved over a period of 4 to 6 weeks postoperatively in all cases.

Postoperative radiographic evaluation in this series of cases showed anatomic reduction of the fracture segments to be good in most of the cases.

Malocclusion constitute a slightly higher percentage in open reduction but it also presents less discomfort to the patient and significantly reduces the time patient spends off work [22].In the current study, there was pre-operative occlusion discrepancies in all 20 cases (100\%) and corrected with 7-15 days IMF with elastics postoperatively.

It becomes difficult to determine what factors are important in the development of post-surgical infection after bone plate osteosynthesis of angle fracture. It may be due to fracture instability; type of surgical approach; the amount of surgical disruption of blood supply to the fracture: teeth in the fracture line; removal versus leaving teeth in the fracture; use of compression versus no compression; the delay in treatment; the nutritional status of the patients; their compliance; their oral hygiene; and their abuse of substances [6].

In our study, infection was mild to moderate $(10 \%)$ which was managed with thorough saline irrigation of the surgical site followed by antibiotic therapy postoperatively. In one case of Group I there was recurrent infection at the left angle surgical site and it was managed with plate removal under local anesthesia and antibiotic therapy thereafter for a week. The reason for this was thought to be poor wound care and compromised oral hygiene status of the patient. In the present study none of the subjects of either groups exhibited malunion or nonunion of fracture segments in postoperative radiographs.

Fixation in Group I patients was done using a new design titanium miniplate and there was difficulty in adaptation of the miniplate. In few of the cases of the same group, a trocar was used for transbuccal fixation of the posterior 
screw of distal extension, as intraoral placement was not feasible. This does not correlate with an in vitro study conducted by B.T. Suer et al. [10].

\section{Conclusion:-}

The angle is one of the most common areas for a fracture of the mandible to occur. Treatment of angle fracture has also involved the greatest frequency of complications relative to other simple fractures. Many techniques and approaches for achieving the goals of restoration of anatomic form, maintenance of segment position and bony union have been described. New techniques bring new possibilities but also new questions.

Intraoral approach seems to be most esthetic approach to any mandibular fracture but in regards to mandibular angle fracture, the approach lacks the accessibility and is indicated in favourable non comminuted dentate fractures of mandibular angle.

Extraoral approach, though gives more accessibility to mandibular angle fractures but is associated with marginal mandibular nerve damage, scar, inability to visualize the occlusion simultaneously during reduction and is indicated in cases of comminution, atrophic and unfavourable fractures of the mandibular angle.

In the present study we have used a new design titanium miniplate for fixation of mandibular angle fractures and compared it with conventional 4 hole titanium miniplate. Though the study sample was less, with the results we have got, we would like to conclude that the use of new design titanium miniplate for fixation of mandibular angle fractures is an effective and reliable mode of fixation in cases of non-comminuted, minimally displaced, dentulous fractures. Although the adaptation of the miniplate and placement of posterior screw of the distal extension is quite cumbersome, it exhibited marginally better anatomical reduction of fracture segments in comparison to conventional 4-hole titanium miniplate. Factors like operator experience; preoperative selection of case and use of trocar in some cases for transbuccal fixation should be considered.

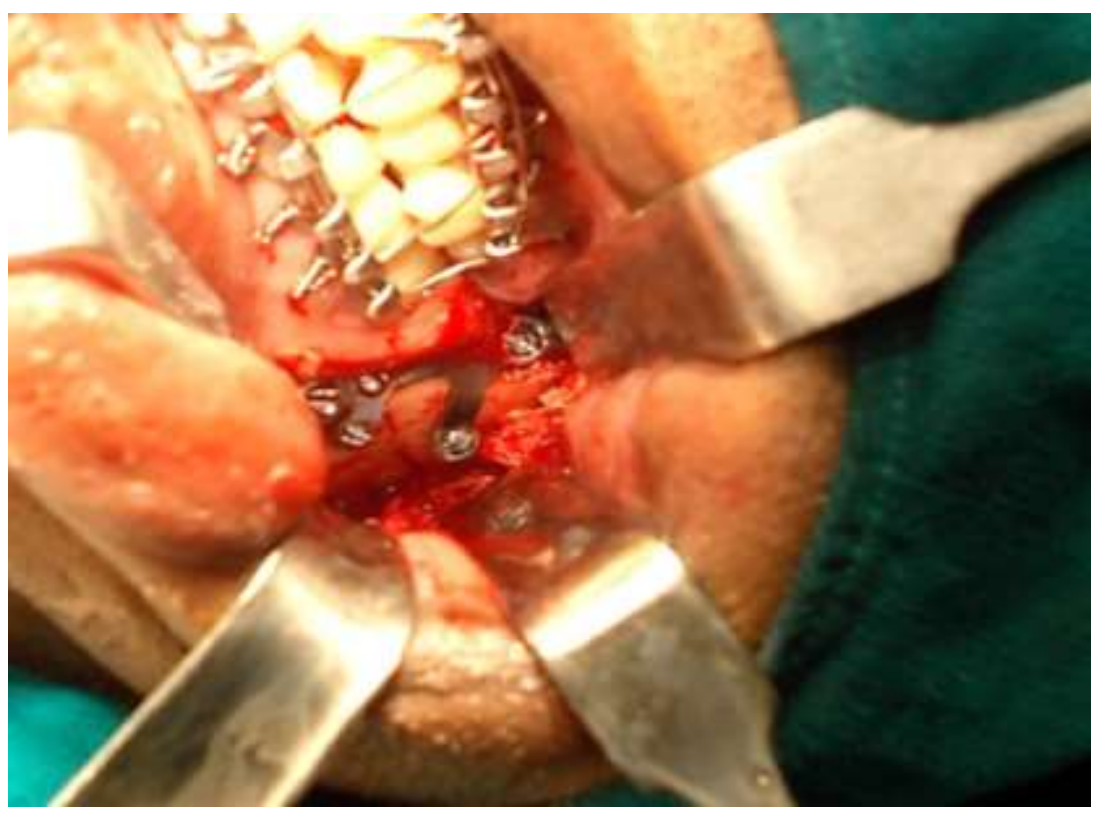

Fig. 1:- Intraoral fixation with New Design titanium miniplate 


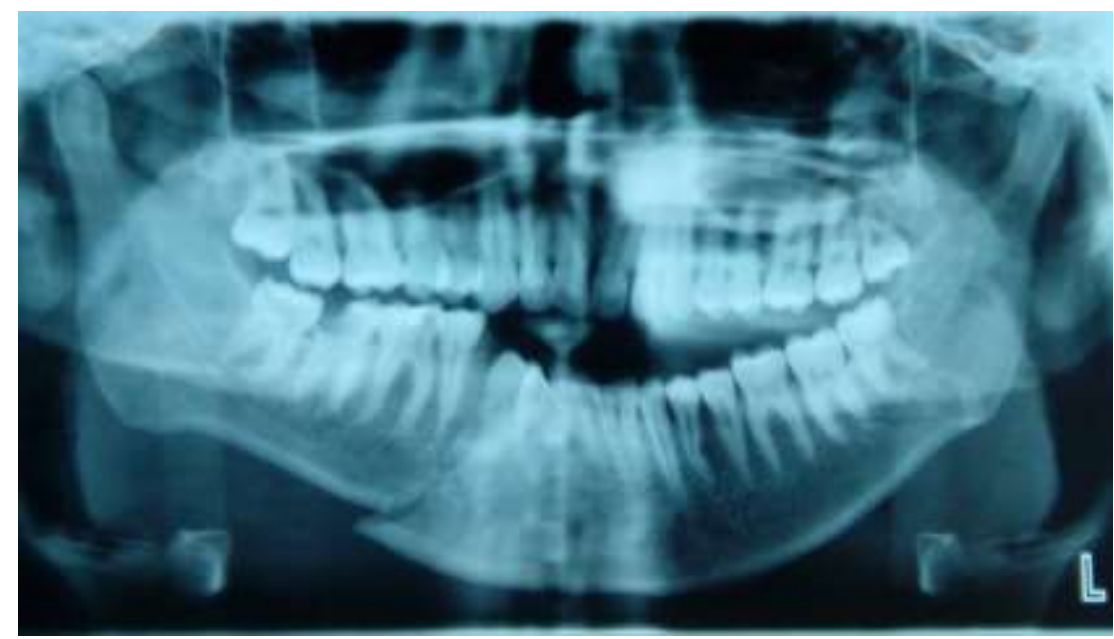

Fig. 2:- Preoperative OPG

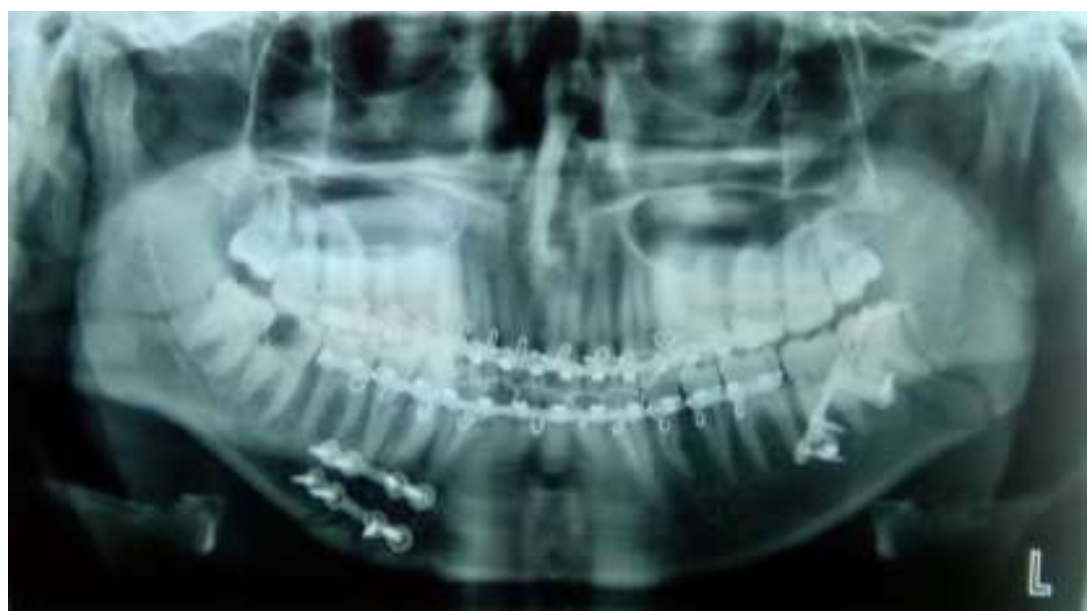

Fig. 3:- Postoperative OPG

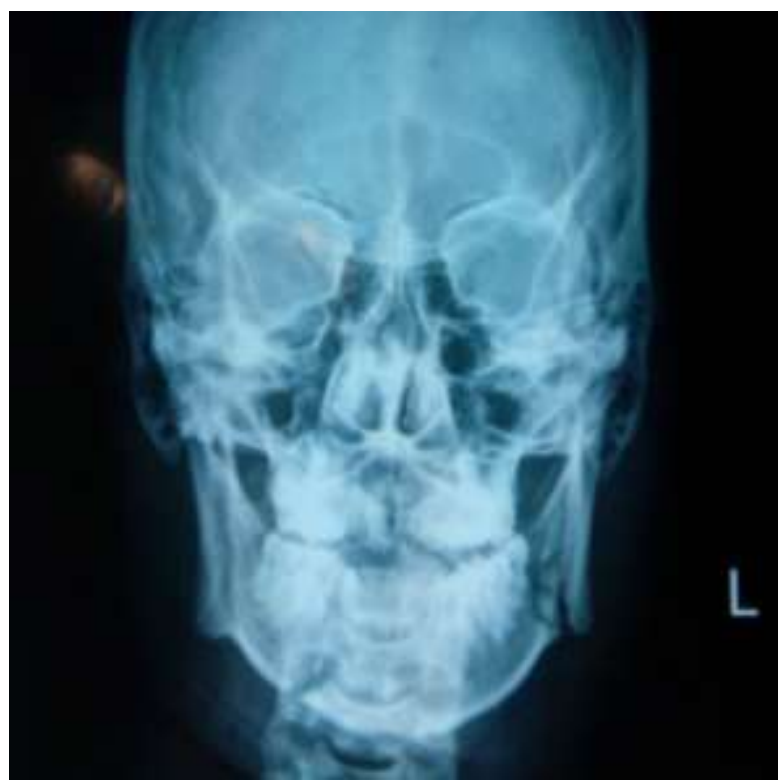

Fig. 4:- Preoperative PA view of mandible 


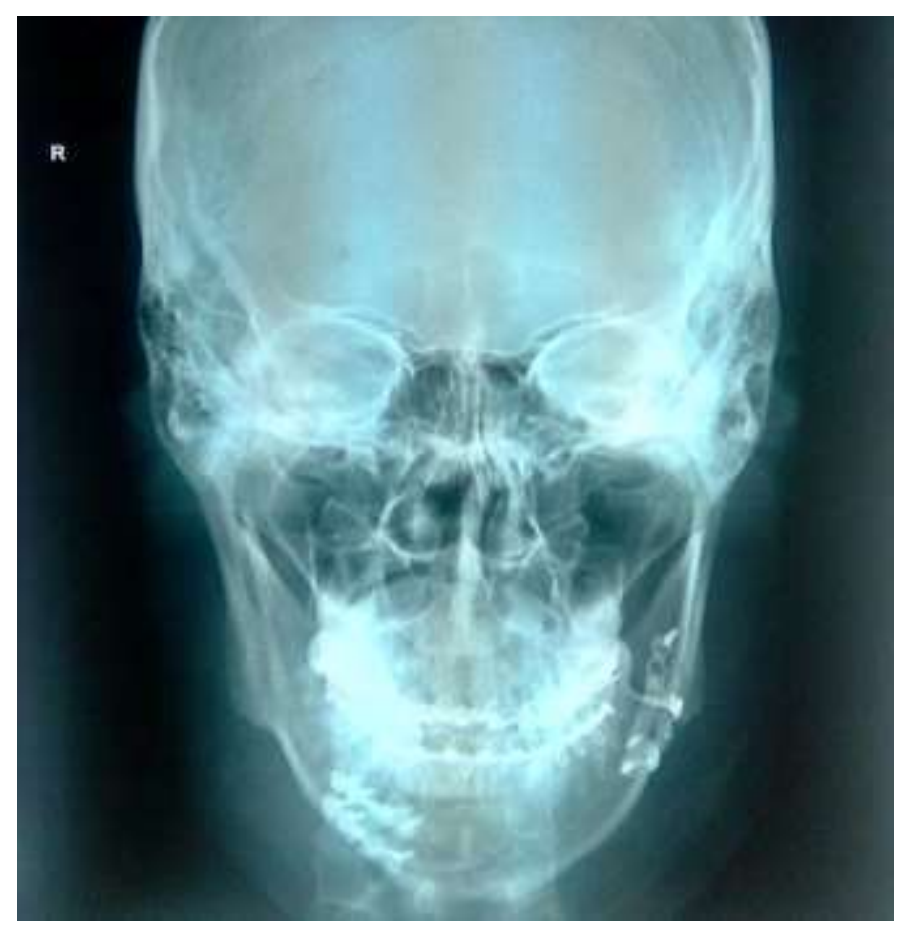

Fig. 5:- Postoperative PA view of mandible

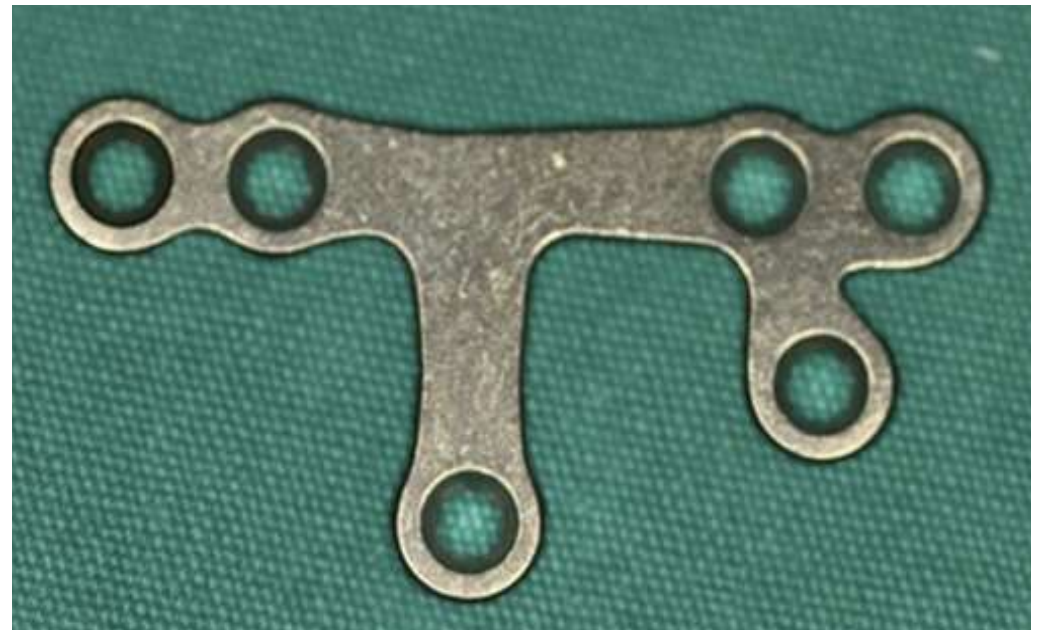

Fig. 6:- New Design titanium miniplate for right side 


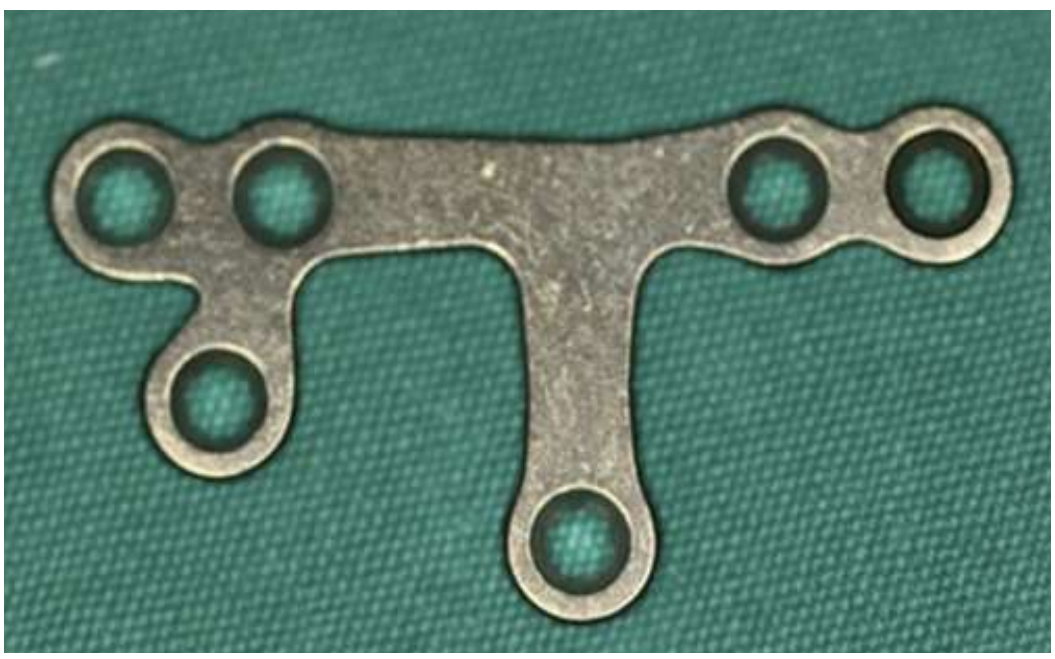

Fig. 7:- New Design titanium miniplate for left side

\section{References:-}

1. Thomas Indresano et al (1994). Principles of Oral and Maxillofacial Surgery. J.B. Lippincot company, Philadelphia, Vol. I (pp. 407-468).

2. John. D. Langdon and Mohan F. Patel (1998). Mandibular osteosynthesis. Chapman and Hall Medical (pp. 339-346).

3. Fonseca Raymond J and Robert V. Walter (1997). Oral and Maxillofacial Trauma. Pennsylvania; W.B. Saunders company. 2nd Edition, Vol. 1 (pp. 473-522).

4. Banks Peter (1993). Killey's Fracture of the Mandible, Vergese Publishing House. 4th Edtion.

5. E. Ellis III. Treatment Methods for Fractures of Mandibular Angle. Int J Oral Maxillofac Surg. 1999;28:243252.

6. Edward Ellis III, Lee Walker. Treatment of mandibular angle fracture using two noncompressionminiplates. J Oral Maxillofac Surg. 1994;52:1032-1036.

7. Edward Ellis III, Lee R.Walker. Treatment of mandibular angle fracture using one non compression miniplate. J Oral Maxillofac Surg. 1996;54:864-871.

8. Andrew J. L. Gear, Elena Apasova, John P. Schmitz and Waren Shubert. Treatment modalities for mandibular angle fractures. 2005;63:655-663.

9. J. P. Hayter, J.I. Cawood. The functional case for miniplates in maxillofacial surgery. Int J Oral Maxillofacial Surg. 1993;22:91-96.

10. B.T Suer et al. Biomechanical evaluation of a new design titanium miniplate for treatment of mandibular angle fractures. Int J Oral Maxillofac Surg. 2014;43:841-845.

11. Anil Kumar Danda. Comparision of a Single NoncompressionMiniplate Versus 2 NoncompressionMiniplates in Treatment of Mandibular Angle Fractures: A Prospective, Randomized Clinical Trial. J of Oral Maxillofac Surg. 2010;68:1565-1567.

12. Digumarthi H: Poster 109: Mandibular angle fractures, single versus two-plate fixation; UAB experience. J Oral Maxillofac Surg. 2012;70:109.

13. Braasch DC, Abubaker AO. Management of mandibular angle fracture. Oral MaxillofacSurgClin North Am. 2013; 25:591.

14. Wald RM, Abemayor E, Zempleny J: The transoral treatment of mandibular fractures using noncompressionminiplates: A prospective study. Ann Plast Surg. 1988; 20:409.

15. Iizuka $\mathrm{T}$, Lindqvist $\mathrm{C}$, Hallikainen $\mathrm{D}$, et al: Infection after rigid internal fixation of mandibular fractures: $\mathrm{A}$ clinical and radiologic study. J Oral Maxillofac Surg. 1991;49:585.

16. Tateyukilizuka, Christian Lindquist Sensory disturbances associated with rigid internal fixation of mandibular fractures. J.Oral.Maxillofac. Surg, 199;49:1264- 1268.

17. Jose Marchena M et al. Sensory abnormalities associated with mandibular fractures: incidence and natural history. J.OralMaxillofacSurg 1998;56:822-825.

18. Sorel Bertrand. Open versus closed reduction of mandibular fractures. Oral and Maxillofacial Surgery Clinics of North America 1998;10:541-565. 
19. Luis A. Passeri, Edward Ellis III, and Douglas P. Sinn. Complications of non- rigid fixation of mandibular angle fractures. J Oral MaxillofacSurg 1993;51: 381-384.

20. Allan B.P and Daly C.G Fractures of the mandible a 35 year retrospective study. Int.J.Oral Maxillofac.Surg.1990; 19:268-271.

21. Lars Lindahl. Condylar fractures of the mandible. IntJ.Oral Surg.1977;6:12-21.

22. Brown J.S and Grew N. Intermaxillary fixation compared to miniplateosteosynthesis in the management of the fractured mandible: An Audit. British Journal of Oral and Maxillofac.Surg.1991;29:308-311.

23. Assael L.A. Treatment of mandibular angle fractures. Plate and screw fixation. J Oral Maxillofacial Surg. 1994;52:757-761.

24. Souyris.F, Lamarche.J.P, Mirfakhrai.A.M. Treatment of mandibular fractures by intraoral placement of bone plates. Journal of Oral Surgery 1980;38:33-35. 